\title{
外源激素处理对三峡消落带落羽杉扦插生根的影响
}

\author{
陈春桦 ${ }^{1,2}$ ，陈雪梅 ${ }^{1,2}$ ，杨治华 ${ }^{1,2}$ ，袁中勋 ${ }^{1,2}$ ，李昌晓 ${ }^{1,2, *}$ \\ 1 西南大学生命科学学院, 三峡库区生态环境教育重点实验室, 重庆市三峡库区植物生态与资源重点实验室, 重庆 400715 \\ 2 西南山地生态循环农业国家级培育基地, 重庆 400715
}

摘要: 为优选落羽杉大龄母树插穗的扦插繁殖技术, 以期培育大量的落羽杉良种壮苗满足三峡消落带植被修复的需要, 采用正 交试验设计, 探究不同外源激素、浓度以及处理时间对三峡消落带落羽杉大龄母树插穗扦插生根的影响,运用隶属函数法对各 处理生根情况进行综合评价。结果表明: (1) 落羽杉插穗皮部和愈伤组织处均有不定根伸出, 两处不定根数量分别占不定根总 数的 $63.9 \%$ 和 $36.1 \%$; (2) 4 种外源激素中, 吲哚丁酸 (IBA)+芸乙酸 (NAA) (等质量比) 和 IBA 处理的插穗生根效果最好, NAA 处理次之,生根粉 (ABT) 处理效果最差; (3) 5 种浓度 $(50 \mathrm{mg} / \mathrm{L} 、 100 \mathrm{mg} / \mathrm{L} 、 150 \mathrm{mg} / \mathrm{L} 、 200 \mathrm{mg} / \mathrm{L}$ 和 $250 \mathrm{mg} / \mathrm{L}$ ) 之间的处理效果无 显著差异; (4) 4 种处理时间 ( $2 \mathrm{~h} 、 4 \mathrm{~h} 、 6 \mathrm{~h}$ 和 $8 \mathrm{~h}$ ) 中, $4 \mathrm{~h}$ 处理的插穗生根效果最佳; (5) 26 个处理组合中, (IBA+ NAA) $\times$ $150 \mathrm{mg} / \mathrm{L} \times 4 \mathrm{~h}$ 处理组合和 $\mathrm{NAA} \times 250 \mathrm{mg} / \mathrm{L} \times 4 \mathrm{~h}$ 处理组合的平均隶属函数值最高, 分别为 0.83 和 0.82 , 清水对照的平均隶属函 数值最低, 为 0.05 。研究初步验证了皮部生根是落羽杉大龄母树插穗的主要生根方式, 笁选出三峡消落带原位适生落羽杉大龄 母树插穗扦插的两种较佳处理组合为 $(\mathrm{IBA}+\mathrm{NAA}) \times 150 \mathrm{mg} / \mathrm{L} \times 4 \mathrm{~h}$ 和 $\mathrm{NAA} \times 250 \mathrm{mg} / \mathrm{L} \times 4 \mathrm{~h}$ 。

关键词:三峡消落带; 落羽杉; 扞插;外源激素;正交试验

\section{Effects of exogenous hormone on rooting of Taxodium distichum cuttings from the hydro-fluctuation belt of the Three Gorges Reservoir}

\author{
CHEN Chunhua ${ }^{1,2}$, CHEN Xuemei ${ }^{1,2}$, YANG Zhihua ${ }^{1,2}$, YUAN Zhongxun ${ }^{1,2}$, LI Changxiao ${ }^{1,2, *}$ \\ 1 Chongqing Key Laboratory of Plant Ecology and Resources Research in Three Gorges Reservoir Region, Key Laboratory of Eco-environments in Three Gorges \\ Reservoir Region (Ministry of Education), Southwest University, School of Life Sciences, Chongqing 400715, China \\ 2 State Cultivation Base of Eco-agriculture for Southwest Mountainous Land, Chongqing 400715, China
}

\begin{abstract}
To optimize the cutting propagation technology of the Taxodium distichum for cultivating a large number of T. distichum seedlings to meet the needs of vegetation restoration in the fluctuating zone of the Three Gorges Reservoir, an orthogonal experiment design was used to explore the effects of different exogenous hormones, concentrations and treatment time on rooting of cuttings of elder $T$. distichum in the hydro-fluctuation belt of the Three Gorges Reservoir. Besides, the subordination function was used to comprehensively evaluate the rooting condition of the cuttings. Results showed that: (1) The number of adventitious roots protruding from the bark and the callus accounted for $63.9 \%$ and $36.1 \%$ of the total number of adventitious roots, respectively; (2) Among these 4 exogenous hormones, IBA+NAA (mass ratio 1:1) and IBA (Indolebutyric acid) had the best effectiveness, followed by NAA (Naphthalene acetic acid), and ABT rooting powder was the poorest; (3) There was no significant difference in facilitating rooting of the cuttings among the five concentrations ( 50 $\mathrm{mg} / \mathrm{L}, 100 \mathrm{mg} / \mathrm{L}, 150 \mathrm{mg} / \mathrm{L}, 200 \mathrm{mg} / \mathrm{L}$ and $250 \mathrm{mg} / \mathrm{L})$; (4) Among these 4 treatment times $(2 \mathrm{~h}, 4 \mathrm{~h}, 6 \mathrm{~h}$ and $8 \mathrm{~h}$ ),

基金项目: 重庆市林业首席专家项目团队项目 ( TD2021-2) ; 重庆市科技兴林项目(2021-9); 中央林业改革发展资金科技推广示范项目(渝林科推 2020-2) ; 重庆市建设科技项目 (城科字 2019-1-4-2)

收稿日期: 2020-09-21; 网络出版日期:2021-07-06

* 通讯作者 Corresponding author.E-mail: lichangx@ swu.edu.cn
\end{abstract}

http : //www.ecologica.cn 
the treatment time of $4 \mathrm{~h}$ had the best effectiveness; ( 5) Among these 26 treatment combinations, ( IBA+NAA) $\times 150 \mathrm{mg} / \mathrm{L}$ $\times 4 \mathrm{~h}$ and $\mathrm{NAA} \times 250 \mathrm{mg} / \mathrm{L} \times 4 \mathrm{~h}$ had the highest average subordinate function value of 0.83 and 0.82 , respectively; and the control group had the lowest average subordinate function value of 0.05 . In this study, it was preliminarily verified that bark rooting was the main way of rooting of the cuttings from elder $T$. distichum, and (IBA+NAA) $\times 150 \mathrm{mg} / \mathrm{L} \times 4 \mathrm{~h} \mathrm{and} \mathrm{NAA \times}$ $250 \mathrm{mg} / \mathrm{L} \times 4 \mathrm{~h}$ were suitable treatments for cutting propagation of elder $T$. distichum in the hydro-fluctuation belt of the Three Gorges Reservoir.

Key Words: The hydro-fluctuation belt of the Three Gorges Reservoir; Taxodium distichum; cuttage propagation; exogenous hormones; orthogonal experiment design

三峡水库建成并运行后, 由于冬蓄夏排的水位调度方式, 形成了水位落差 $30 \mathrm{~m}$ 、面积近 $400 \mathrm{~km}^{2}$ 的消落 带 ${ }^{[1]}$ 。三峡库区消落带垂直落差大、淹水时间长、季节性水淹等特点, 使得库区植被遭到破坏, 土壤侵蚀强 烈, 生态系统受到严重干扰 ${ }^{[2-3]}$ 。植被作为消落带生态服务功能的提供主体, 具有增强边坡稳定性、净化水 质、维持和增强消落带生物生产力和生物多样性等诸多功能 ${ }^{[4]}$ 。因此, 如何重建消落带植被已成为研究关注 的热点问题。目前, 已成功笁选出了一些具有较强耐淹性的乔木树种, 如落羽杉 (Taxodium distichum) ${ }^{[5]}$ 、池

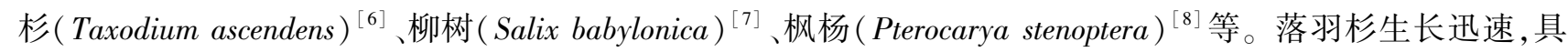
有较强的耐淹性和耐旱性, 在历经多年反复水淹后仍表现出较好的生长适应性, 被列为三峡库区消落带人工 植被重建的优良物种。落羽杉为杉科落羽杉属 (Taxodium Rich.) 落叶乔木, 原产于北美东南部, 现已广泛引种 到世界各地, 我国江苏、浙江、安徽、湖北、江西、福建、四川、河南、山东和陕西等省均有大面积人工林 ${ }^{[9]}$ 。目 前, 用于三峡库区消落带植被修复与重建的落羽杉主要是从外地调运的实生苗, 使得栽种成本增加, 且调运的 苗木病虫害偏多, 不一定适应当地的微环境和小气候。因此, 落羽杉适应性繁殖技术的研究对三峡库区消落 带植被的修复与重建具有重要意义。

落羽杉不同种源和个体对多种胁迫的耐受力存在差异, 因此选择高抗性个体进行造林是十分必要和可行 的 ${ }^{[10]}$ 。落羽杉种子具有休眠现象, 自然条件下发芽率很低 ${ }^{[11-12]}$, 而扦插繁殖作为无性繁殖的一种方式, 与播 种繁殖相比, 能够很好地保留母体优良性状, 此外还具有繁殖速度快、成本低、取材方便、操作方法简单等优 点 ${ }^{[13-14]}$ 。截至 2018 年, 忠县汝溪河消落带植被修复示范基地的落羽杉林经历了 $6 \mathrm{a}$ 的反复水淹, 仍表现出良 好的生长特性 ${ }^{[15]}$ 。因此, 有必要以该落羽杉林为插穗来源, 对其进行扞插繁殖技术的研究。目前, 国内外对 落羽杉扦插繁殖技术的研究主要集中在外源激素的选择、基质、插穗的质量和外源激素的使用方法等方面,研 究多以 1-2 a 生实生苗枝条为扦插材料,插穗经激素处理后的生根效果显著提高 ${ }^{[16-21]}$ 。吴落军 ${ }^{[19]}$ 系统地探 究了不同外源激素种类、外源激素浓度及处理时间对落羽杉 $1 \mathrm{a}$ 生实生苗萌生条扦插的影响,结果表明, 这三 种因素均对插穗生根效果产生显著影响,但浓度和处理时间的设置上仍有进一步优化的空间。目前, 对于落 羽杉大龄母树枝条的扞插技术还缺乏系统研究,因此,本研究将在上述已有研究的基础上,采用拟水平正交试 验设计,探究不同外源激素种类、激素浓度及处理时间对三峡库区消落带原位生长的 $9 \mathrm{a}$ 生落羽杉扞插生根 的影响, 并采用隶属函数法, 对各生根指标进行综合评价。本研究旨在篮选出最佳激素、浓度与时间的处理组 合, 以期提高消落带落羽杉的扞插繁殖技术, 培育大量优质的落羽杉幼苗以满足三峡库区消落带植被修复重 建的需要。

\section{1 材料与方法}

\section{1 插床的准备}

插床位于西南大学三峡库区生态环境教育部重点实验室实验基地的简易大棚内。扦插基质为草炭+珍 珠岩+紫色土(3:3:1), 基质厚度为 $20 \mathrm{~cm}$ 。扦插前 $7 \mathrm{~d}$ 用 $5 \%$ 的多菌灵对土壤进行消毒, 并用塑料薄膜覆盖, 扞 插前一天揭开备用。 


\section{2 插穗的采集与制备}

2018 年 9 月下旬在重庆市忠县石宝镇共和村汝溪河流域的三峡消落带植被恢复试验示范基地, 以 165一 $175 \mathrm{~m}$ 海拔之间的 $9 \mathrm{a}$ 生落羽杉实生苗为采穗母树,选择树体中上部生长健壮、无病虫害、无机械损伤的半木 质化枝条制备插穗。枝条在阴天采集, 及时喷水保湿,并送回实验室制备插穗。穗长 $10-12 \mathrm{~cm}$, 保留 3-4 个叶片,上端口于着生羽状叶或脱落性小枝上方 $0.5-1.0 \mathrm{~cm}$ 处平截,下端口于着生羽状叶或侧枝下方 $0.5 \mathrm{~cm}$ 处单面斜切,并剪除插穗下部的叶片或侧枝。将来自不同海拔的插穗按比例混合均匀,30 株一捆。

\section{3 试验设计}

试验于 2018 年 9 月下旬进行。采用 L25 $\left(5^{6}\right)$ 正交表进行拟水平法的正交试验设计, 以清水浸泡处理作 为对照, 探讨外源激素种类、激素浓度和处理时间对落羽杉扦插生根的影响。试验共 26 个处理组合, 每个处 理 3 个小区, 即 3 个重复, 每个小区 30 株插穗。拟水平正交设计因素和水平见表 1 。

表 1 落羽杉扞插正交试验因素及水平

Table 1 Factors and horizontal arrangement in orthogonal experiment of cutting propagation of Taxodium distichum

\begin{tabular}{clcc}
\hline \multirow{2}{*}{$\begin{array}{c}\text { 水平 } \\
\text { Level }\end{array}$} & \multicolumn{1}{c}{\begin{tabular}{c} 
因素 Factor \\
外源激素 \\
\cline { 2 - 4 }
\end{tabular}} & $\begin{array}{c}\text { 浓度/ }(\mathrm{mg} / \mathrm{L}) \\
\text { Concentration }\end{array}$ & $\begin{array}{c}\text { 处理时间 } / \mathrm{h} \\
\text { Treatment time }\end{array}$ \\
\hline 1 & IBA & 50 & 2 \\
2 & NAA & 100 & 4 \\
3 & ABT & 150 & 6 \\
4 & IBA+NAA (1:1) & 200 & 4 \\
5 & IBA+NAA (1:1) & 250 & 4 \\
\hline
\end{tabular}

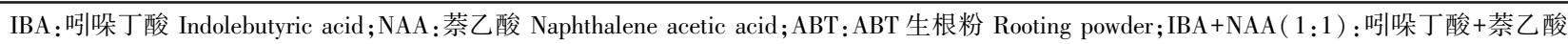
(等质量比) Indolebutyric acid+Naphthalene acetic acid(mass ratio 1:1)

\section{4 扞插方法及扦插后管理}

插穗基部 $2 \mathrm{~cm}$ 浸泡于外源激素溶液中,扦插前用细木棍在基质上打孔,株行距 $4 \mathrm{~cm} \times 5 \mathrm{~cm}$ 扦插深度为 5-6 cm, 扞插后用两手按实苗床,使插穗与插壤密接,随即浇透水。根据天气情况及时调整浇水时间, 并及 时除草。通过遮阳网将遮阳率控制在 $60 \%$, 扦插一个月后撤去遮阳网。

\section{5 指标调查及数据分析}

2019 年 6 月下旬对各处理插穗的存活率、生根率、平均根数、总根长、根表面积以及根体积进行调查统 计。存活率=存活株数/扞插总株数 $\times 100 \%$; 生根率=生根株数 $/$ 扞插总株数 $\times 100 \%$; 平均根数 $=$ 不定根总数 $/$ 生根株数。数字化扫描仪 (STD1600 Epson USA) 扫描根系, win. Rhzo (Version 410B) 根系分析系统软件 ( Refent Instmment Inc,Canada) 对所有生根插穗总根长、根表面积和根总体积进行定量分析。

运用 SPSS 22.0 对所有指标进行方差分析,Duncan 检验法进行多重比较。为了对各处理生根情况进行综 合评价, 对所有指标进行隶属函数值计算, 隶属函数计算公式为:

$$
U\left(X_{j}\right)=\left(X_{j}-X_{\min }\right) /\left(X_{\max }-X_{\min }\right)
$$

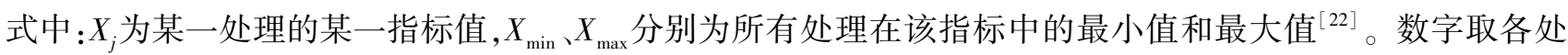
理下不同测定指标隶属函数值的平均值作为评价标准, 隶属函数值越大, 扞插效果越好。

\section{2 研究结果}

\section{1 落羽杉嫩枝扦插的生根类型}

本研究中, 落羽杉插穗皮部和愈伤组织处均有不定根长出。实验共有 2340 株插穗, 其中产生愈伤组织的 插穗有 1205 株,生根插穗共有 345 株, 其中仅在皮部产生不定根的有 206 株, 占总生根插穗的 $59.7 \%$; 仅在愈 伤组织处产生不定根的有 69 株, 占总生根插穗的 $20.0 \%$; 在皮部和愈伤组织处均有不定根产生的有 70 株, 占 
总生根插穗的 $20.3 \%$ 。生根插穗共产生 593 条不定根, 其中皮部处的有 379 条, 占不定根总数的 $63.9 \%$, 愈伤 组织处有 214 条, 占不定根总数的 $36.1 \%$ 。

2.2 正交试验结果方差分析

落羽杉扞插正交试验 6 个生根指标的方差分析结果 (表 2) 表明,外源激素种类对生根率、存活率、总根 长、根表面积以及根体积的影响达到极显著水平 $(P<0.01)$, 对根数的影响不显著 $(P>0.05)$; 外源激素浓度对 所有指标均无显著影响 $(P>0.05)$; 外源激素处理时间对存活率、总根长和根表面积有极显著影响 $(P<0.01)$, 对生根率和根体积有显著影响 $(P<0.05)$, 对根数的影响不显著 $(P>0.05)$ 。由 $F$ 值的大小可知, 三个因素对 六个生根指标的影响主次顺序为外源激素种类 $>$ 处理时间 $>$ 外源激素浓度, 即外源激素种类的影响最大, 其次 是处理时间和生长素浓度。

表 2 外源激素种类、浓度和处理时间对落羽杉扦插生根影响的方差分析

Table 2 Variance analysis of the effects of different kinds of exogenous hormones, concentrations and treatment time on cutting propagation of Taxodium distichum

\begin{tabular}{|c|c|c|c|c|c|c|c|c|c|c|c|c|}
\hline \multirow{2}{*}{$\begin{array}{l}\text { 变量 } \\
\text { Variables }\end{array}$} & \multicolumn{2}{|c|}{$\begin{array}{c}\text { 生根率/\% } \\
\text { Rooting percentage }\end{array}$} & \multicolumn{2}{|c|}{$\begin{array}{l}\text { 存活率/\% } \\
\text { Survival rate }\end{array}$} & \multicolumn{2}{|c|}{$\begin{array}{c}\text { 根数 } \\
\text { Number of roots }\end{array}$} & \multicolumn{2}{|c|}{$\begin{array}{c}\text { 总根长/cm } \\
\text { Total root length }\end{array}$} & \multicolumn{2}{|c|}{$\begin{array}{l}\text { 根表面积 } / \mathrm{cm}^{2} \\
\text { Root surface area }\end{array}$} & \multicolumn{2}{|c|}{$\begin{array}{c}\text { 根总体积 } / \mathrm{cm}^{3} \\
\text { Root volume }\end{array}$} \\
\hline & $F$ & $P$ & $F$ & $P$ & $F$ & $P$ & $F$ & $P$ & $F$ & $P$ & $F$ & $P$ \\
\hline $\begin{array}{l}\text { 外源激素 } \\
\text { Exogenouse hormone }\end{array}$ & 10.44 & 0.001 & 6.88 & 0.004 & 7.22 & 0.004 & 16.27 & 0.000 & 13.05 & 0.000 & 11.01 & 0.001 \\
\hline $\begin{array}{l}\text { 浓度 } \\
\text { Concentration }\end{array}$ & 1.17 & 0.366 & 1.07 & 0.409 & 0.36 & 0.835 & 1.29 & 0.322 & 0.96 & 0.462 & 1.62 & 0.225 \\
\hline $\begin{array}{l}\text { 处理时间 } \\
\text { Treatment time }\end{array}$ & 5.36 & 0.011 & 5.91 & 0.008 & 1.82 & 0.190 & 7.89 & 0.003 & 6.22 & 0.007 & 3.62 & 0.04 \\
\hline
\end{tabular}

\section{3 外源激素种类对落羽杉扞插生根性状的影响}

不同外源激素间生根指标的 Duncan 多重比较结果 (表 3) 表明,在生根率、根表面积和根体积方面, ABT 的表现最差, 分别为 $4.67 \% 、 3.77 \mathrm{~cm}^{2} 、 0.067 \mathrm{~cm}^{3}$, IBA、NAA、IBA+NAA 三个处理的表现差异不显著 $(P>0.05)$; 在存活率方面, IBA+NAA 处理和 IBA 处理的表现最佳,存活率分别为 $88.56 \%$ 和 $85.56 \%$,显著高于 ABT 处理 $(81.33 \%)$, 但与 NAA 处理 $(84.67 \%)$ 的差异不显著 $(P>0.05)$, ABT 处理与 NAA 处理间的差异也不显著 $(P>$ $0.05)$; 在总根长方面, IBA+NAA 处理和 IBA 处理的表现最佳, 分别为 $35.41 \mathrm{~cm}$ 和 $34.11 \mathrm{~cm}$, NAA 处理 $(23.53$ $\mathrm{cm})$ 次之, $\mathrm{ABT}$ 处理效果 $(12.66 \mathrm{~cm})$ 最差。综合来看, 使用 IBA+NAA 处理或 IBA 处理生根效果最好, ABT 处 理的生根效果最差。

表 3 不同外源激素处理下扦插生根性状的多重比较

Table 3 Multiple comparison of rooting trait of cuttings treated by different exogenous hormones

\begin{tabular}{lcccc}
\hline $\begin{array}{l}\text { 外源激素 } \\
\text { Exogenous hormone }\end{array}$ & $\begin{array}{c}\text { 生根率 } \% \\
\text { Rooting percentage }\end{array}$ & $\begin{array}{c}\text { 存活率 } / \% \\
\text { Survival rate }\end{array}$ & $\begin{array}{c}\text { 总根长 } / \mathrm{cm} \\
\text { Total root length }\end{array}$ & $\begin{array}{c}\text { 根表面积 } / \mathrm{cm}^{2} \\
\text { Root surface area }\end{array}$ \\
\hline IBA & $18.44 \mathrm{a}$ & $85.56 \mathrm{a}$ & $34.11 \mathrm{a}$ & $8.83 \mathrm{a}$ \\
NAA & $16.00 \mathrm{a}$ & $84.67 \mathrm{ab}$ & $23.53 \mathrm{~b}$ & $8.27 \mathrm{a}$ \\
ABT & $4.67 \mathrm{~b}$ & $81.33 \mathrm{~b}$ & $12.66 \mathrm{c}$ & $3.77 \mathrm{~b}$ \\
IBA+NAA $(1: 1)$ & $18.45 \mathrm{a}$ & $88.56 \mathrm{a}$ & $35.41 \mathrm{a}$ & $0.204 \mathrm{a}$ \\
\hline
\end{tabular}

同一列不同小写字母表示处理间有显著差异 $(P<0.05)$

\section{4 外源激素浓度对落羽杉扞插生根性状的影响}

根据正交试验方差分析结果 (表 2),5 种外源激素浓度处理 (50 mg/L、100 mg/L、150 mg/L、200 mg/L、 $250 \mathrm{mg} / \mathrm{L})$ 对落羽杉插穗各生根指标均无显著影响 $(P>0.05)$ 。

2.5 外源激素处理时间对落羽杉扞插生根性状的影响

Duncan 多重比较结果(表 4) 表明, 在生根率方面, $4 \mathrm{~h}$ 处理的表现最好, 为 $19.78 \%$, 显著高于其他处理, 
$2 \mathrm{~h} 、 6 \mathrm{~h}$ 和 $8 \mathrm{~h}$ 这三个处理间生根率并无显著差异 $(P>0.05)$; 在存活率方面, 表现最好的是 $4 \mathrm{~h}$ 处理, 其存活 率显著高于 $2 \mathrm{~h}$ 处理和 $6 \mathrm{~h}$ 处理, 但与 $8 \mathrm{~h}$ 处理的差异不显著 $(P>0.05), 6 \mathrm{~h}$ 处理和 $8 \mathrm{~h}$ 处理之间的差异也不显 著 $(P>0.05)$; 在总根长方面, $4 \mathrm{~h}$ 处理的表现最好, 显著高于 $6 \mathrm{~h}$ 和 $8 \mathrm{~h}$ 处理, 与 $2 \mathrm{~h}$ 处理的差异不显著 $(P>$ $0.05)$; 在根表面积和根体积方面, 表现最好的是 $4 \mathrm{~h}$ 处理,显著高于 $6 \mathrm{~h}$ 处理,但与 $2 \mathrm{~h}$ 处理和 $8 \mathrm{~h}$ 处理间的差 异不显著 $(P>0.05)$ 。综上, 随着浸泡时间的延长, 落羽杉插穗的各生根性状大致呈现先升高后降低的趋势, 浸泡时间为 $4 \mathrm{~h}$ 时插穗各生根性状均为最好。

表 4 不同处理时间下扦插生根性状的多重比较

Table 4 Multiple comparison of rooting trait of cuttings treated by different treatment time

\begin{tabular}{|c|c|c|c|c|c|}
\hline 处理时间/h & 生根率/\% & 存活率/\% & 总根长/cm & 根表面积/cm² & 根体积 $/ \mathrm{cm}^{3}$ \\
\hline Treatment time & Rooting percentage & Survival rate & Total root length & Root surface area & Root volume \\
\hline 2 & $12.00 \mathrm{~b}$ & $83.11 b$ & $28.08 \mathrm{ab}$ & $8.16 \mathrm{ab}$ & $0.170 \mathrm{ab}$ \\
\hline 4 & $19.78 \mathrm{a}$ & $88.67 \mathrm{a}$ & $34.94 \mathrm{a}$ & $10.09 \mathrm{a}$ & $0.229 \mathrm{a}$ \\
\hline 6 & $10.89 \mathrm{~b}$ & $83.11 b$ & $18.69 \mathrm{c}$ & $5.67 \mathrm{~b}$ & $0.136 b$ \\
\hline 8 & $13.55 \mathrm{~b}$ & $85.11 \mathrm{ab}$ & $24.47 b c$ & $7.58 \mathrm{ab}$ & $0.176 \mathrm{ab}$ \\
\hline
\end{tabular}

同一列不同小写字母表示处理间有显著差异 $(P<0.05)$

2.6 各处理组合下落羽杉扞插生根指标的综合评价

采用模糊数学中的隶属函数法, 对 26 个处理组合的扞插生根指标进行综合评价。根据隶属函数公式 $U\left(X_{j}\right)=\left(X_{j}-X_{\text {min }}\right) /\left(X_{\text {max }}-X_{\text {min }}\right)$ 分别计算出各处理下生根指标的隶属函数值, 将 6 个指标的隶属函数值进 行平均得到各处理的平均隶属函数值, 平均隶属函数值越大, 表明该处理条件下落羽杉插穗扦插生根的效果 越好。由表 5 可知, $150 \mathrm{mg} / \mathrm{L} \mathrm{IBA}+\mathrm{NAA}$ 处理 $4 \mathrm{~h}$ 的生根效果最好, 其平均隶属函数值最大, 为 0.83 , 根表面积 $\left(13.63 \mathrm{~cm}^{2}\right)$ 在所有组合中最大, 生根率 $(24.44 \%)$ 、存活率 $(92.22 \%)$ 、总根长 $(43.75 \mathrm{~cm})$ 和根体积 $\left(0.33 \mathrm{~cm}^{3}\right)$ 也都位列前三; $250 \mathrm{mg} / \mathrm{L} \mathrm{NAA}$ 处理 $4 \mathrm{~h}$ 的生根效果次之, 其平均隶属函数值为 0.82 , 生根率 $(31.11 \%)$ 、存活率 $(94.44 \%)$ 、根体积 $\left(0.37 \mathrm{~cm}^{3}\right)$ 在所有组合中最大, 总根长 $(33.06 \mathrm{~cm})$ 、根表面积 $\left(12.52 \mathrm{~cm}^{2}\right)$ 的表现也较好; 清 水对照处理的生根效果最差, 其平均隶属函数值为最小, 为 0.05 , 存活率 $(77.78 \%)$ 、根表面积 $\left(2.77 \mathrm{~cm}^{2}\right)$ 和根 体积 $\left(0.03 \mathrm{~cm}^{3}\right)$ 在所有组合中表现最差,生根率 $(4.44 \%)$ 、根数 $(1.25)$ 、总根长 $(10.35 \mathrm{~cm})$ 的表现也较差。因 此,落羽杉扦插生根的最优组合为 $150 \mathrm{mg} / \mathrm{L}$ IBA+NAA 处理 $4 \mathrm{~h}$ 。

\section{3 讨论}

不定根能否形成决定着植物扦插苗能否成活。根据不定根发生过程中的插穗外部形态, 可将生根类型分 为皮部生根型、愈伤生根型和混合生根型 ${ }^{[23]}$ 。多种生根类型的树种比单一生根类型的树种有更多的生根部 位,在其他外界环境条件相同的情况下,更容易促进不定根的形成,扦插成活率就越高 ${ }^{[24]}$ 。本研究发现,落羽 杉插穗皮部和愈伤组织处都有不定根的出现, 这与吴落军 ${ }^{[19]}$ 的研究结果相同,但在形成愈伤组织的 1205 株 插穗里, 仅有 139 株在愈伤组织处长出不定根。郭素娟 ${ }^{25]}$ 认为, 对大多数树种来说, 愈伤组织的形成和不定 根的形成并不存在直接的相关性, 不定根从愈伤组织中伸出, 但其愈伤组织中没有根原基的发生,两者同时产 生的原因可能是它们需要的内外条件相同。愈伤组织可防止细菌侵人和插穗的物质流失, 还可以充当营养物 质及水分等的运输桥梁, 但如果愈伤组织过大,则会消耗插条内过多的生根营养物质而老化, 反而导致插条不 能生根 ${ }^{[26-28]}$ 。本研究只能初步验证落羽杉扦插以皮部生根为主, 三种生根类型均有, 但其生根类型的确认还 需要进一步的解剖学观察。

植物扦插生根是一个复杂的生理、生态及解剖学过程,受植物生物学特性、生理年龄、插穗木质化程度、外 源激素的使用、扦插季节及基质的综合影响 ${ }^{[25,29-30]}$ 。在影响扦插生根率的外部因素中, 植物生长调节剂起着 比较重要的作用 ${ }^{[31]}$ 。外源激素可通过调节插穗内部与不定根生成相关的内源激素和多种酶类,进而促进插 穗生根 ${ }^{[32-35]}$ 。本研究发现外源激素种类是对落羽杉扦插的影响作用最大的因子,4 种外源激素中, IBA+NAA 
表 5 各处理组合对落羽杉扞插生根指标的影响及隶属函数评价

Table 5 Effects of each treatment combination on rooting trait of Taxodium distichum and membership function evaluation

\begin{tabular}{|c|c|c|c|c|c|c|c|}
\hline $\begin{array}{l}\text { 处理 } \\
\text { Treatment }\end{array}$ & $\begin{array}{c}\text { 生根率/\% } \\
\text { Rooting } \\
\text { percentage }\end{array}$ & $\begin{array}{c}\text { 存活率/\% } \\
\text { Survival } \\
\text { rate }\end{array}$ & $\begin{array}{c}\text { 根数 } \\
\text { Number } \\
\text { of roots }\end{array}$ & $\begin{array}{c}\text { 总根长/cm } \\
\text { Total root } \\
\text { length }\end{array}$ & $\begin{array}{c}\text { 根表面积 } / \mathrm{cm}^{2} \\
\text { Root surface } \\
\text { area }\end{array}$ & $\begin{array}{c}\text { 根总体积 } / \mathrm{cm}^{3} \\
\text { Root volume }\end{array}$ & $\begin{array}{c}\text { 平均隶属 } \\
\text { 函数值 } \mathrm{U}(\mathrm{X}) \\
\text { Average value of } \\
\text { subordinate function }\end{array}$ \\
\hline $\mathrm{IBA} \times 250 \mathrm{mg} / \mathrm{L} \times 2 \mathrm{~h}$ & 21.11 & 85.56 & 1.67 & 27.93 & 8.65 & 0.19 & 0.51 \\
\hline $\mathrm{IBA} \times 100 \mathrm{mg} / \mathrm{L} \times 4 \mathrm{~h}$ & 25.56 & 85.56 & 1.52 & 44.80 & 12.28 & 0.27 & 0.69 \\
\hline $\mathrm{IBA} \times 200 \mathrm{mg} / \mathrm{L} \times 4 \mathrm{~h}$ & 20 & 90.00 & 1.83 & 47.60 & 11.22 & 0.21 & 0.69 \\
\hline $\mathrm{IBA} \times 150 \mathrm{mg} / \mathrm{L} \times 6 \mathrm{~h}$ & 15.56 & 82.22 & 1.36 & 24.37 & 6.10 & 0.17 & 0.35 \\
\hline $\mathrm{IBA} \times 50 \mathrm{mg} / \mathrm{L} \times 8 \mathrm{~h}$ & 10 & 84.44 & 1.56 & 25.87 & 5.92 & 0.11 & 0.33 \\
\hline $\mathrm{NAA} \times 150 \mathrm{mg} / \mathrm{L} \times 2 \mathrm{~h}$ & 7.78 & 77.78 & 2.43 & 15.45 & 6.48 & 0.14 & 0.32 \\
\hline NAA $\times 50 \mathrm{mg} / \mathrm{L} \times 4 \mathrm{~h}$ & 21.11 & 88.89 & 1.21 & 32.39 & 9.30 & 0.21 & 0.54 \\
\hline $\mathrm{NAA} \times 250 \mathrm{mg} / \mathrm{L} \times 4 \mathrm{~h}$ & 31.11 & 94.44 & 1.61 & 33.06 & 12.52 & 0.37 & 0.82 \\
\hline NAA $\times 200 \mathrm{mg} / \mathrm{L} \times 6 \mathrm{~h}$ & 6.67 & 80.00 & 1.33 & 6.53 & 3.02 & 0.08 & 0.11 \\
\hline NAA $\times 100 \mathrm{mg} / \mathrm{L} \times 8 \mathrm{~h}$ & 13.33 & 82.22 & 1.58 & 30.24 & 10.05 & 0.22 & 0.47 \\
\hline ABT $\times 50 \mathrm{mg} / \mathrm{L} \times 2 \mathrm{~h}$ & 3.33 & 80.00 & 1.67 & 11.02 & 3.54 & 0.06 & 0.14 \\
\hline ABT $\times 200 \mathrm{mg} / \mathrm{L} \times 4 \mathrm{~h}$ & 5.56 & 82.22 & 1.00 & 11.86 & 4.17 & 0.08 & 0.13 \\
\hline ABT $\times 250 \mathrm{mg} / \mathrm{L} \times 4 \mathrm{~h}$ & 8.89 & 85.56 & 1.50 & 18.26 & 4.65 & 0.09 & 0.27 \\
\hline ABT $\times 100 \mathrm{mg} / \mathrm{L} \times 6 \mathrm{~h}$ & 3.33 & 80.00 & 1.00 & 12.24 & 3.32 & 0.05 & 0.07 \\
\hline ABT $\times 150 \mathrm{mg} / \mathrm{L} \times 8 \mathrm{~h}$ & 2.22 & 78.89 & 2.00 & 9.90 & 3.19 & 0.05 & 0.14 \\
\hline$(\mathrm{IBA}+\mathrm{NAA}) \times 100 \mathrm{mg} / \mathrm{L} \times 2 \mathrm{~h}$ & 14.44 & 88.89 & 1.62 & 39.97 & 9.35 & 0.17 & 0.55 \\
\hline$(\mathrm{IBA}+\mathrm{NAA}) \times 50 \mathrm{mg} / \mathrm{L} \times 4 \mathrm{~h}$ & 18.89 & 88.89 & 1.53 & 34.47 & 9.67 & 0.22 & 0.57 \\
\hline$(\mathrm{IBA}+\mathrm{NAA}) \times 150 \mathrm{mg} / \mathrm{L} \times 4 \mathrm{~h}$ & 24.44 & 92.22 & 1.86 & 43.74 & 13.63 & 0.33 & 0.83 \\
\hline$(\mathrm{IBA}+\mathrm{NAA}) \times 250 \mathrm{mg} / \mathrm{L} \times 6 \mathrm{~h}$ & 16.67 & 86.67 & 1.60 & 25.95 & 8.53 & 0.22 & 0.49 \\
\hline$(\mathrm{IBA}+\mathrm{NAA}) \times 200 \mathrm{mg} / \mathrm{L} \times 8 \mathrm{~h}$ & 24.44 & 92.22 & 1.73 & 22.74 & 7.52 & 0.20 & 0.57 \\
\hline$(\mathrm{IBA}+\mathrm{NAA}) \times 200 \mathrm{mg} / \mathrm{L} \times 2 \mathrm{~h}$ & 13.33 & 83.33 & 2.67 & 46.03 & 12.78 & 0.29 & 0.73 \\
\hline$($ IBA+NAA $) \times 100 \mathrm{mg} / \mathrm{L} \times 4 \mathrm{~h}$ & 16.67 & 87.78 & 2.40 & 42.19 & 10.16 & 0.21 & 0.67 \\
\hline$(\mathrm{IBA}+\mathrm{NAA}) \times 150 \mathrm{mg} / \mathrm{L} \times 4 \mathrm{~h}$ & 25.56 & 91.11 & 1.86 & 41.05 & 13.28 & 0.29 & 0.79 \\
\hline$(\mathrm{IBA}+\mathrm{NAA}) \times 50 \mathrm{mg} / \mathrm{L} \times 6 \mathrm{~h}$ & 12.22 & 86.67 & 1.82 & 24.39 & 7.37 & 0.16 & 0.44 \\
\hline$(\mathrm{IBA}+\mathrm{NAA}) \times 250 \mathrm{mg} / \mathrm{L} \times 8 \mathrm{~h}$ & 17.78 & 87.78 & 2.13 & 33.60 & 11.15 & 0.30 & 0.67 \\
\hline CK & 4.44 & 77.78 & 1.25 & 10.35 & 2.77 & 0.03 & 0.05 \\
\hline
\end{tabular}

(1:1) 和 IBA 处理的插穗生根效果最好, NAA 处理次之, ABT 处理效果最差。一般来说,两种及两种以上的激 素混合处理比单一激素处理更具优越性,其生根效果比单一激素好 ${ }^{[36]}$ 。孙旭东等 ${ }^{[37]}$ 研究了不同生根剂对色 木械扦插的影响,结果表明 NAA 和 IBA 混合剂处理效果高于 NAA 和 IBA 单独处理效果。魏黔春 ${ }^{[38]}$ 对侧柏 古树的研究以及刘帅 ${ }^{[39]}$ 对柽柳属树种的研究也得到类似的结果。本研究中, IBA+NAA(1:1) 处理的生根效果 最好,但各项生根指标与 IBA 处理并无显著差异, 这可能与 IBA 和 NAA 之间的配比有关。郑健等 ${ }^{[40]}$ 的研究 发现 IBA+NAA(10:1) 和 IBA+NAA (5:1) 两种处理对花楸扦插的促进效果明显优于 IBA+NAA(2:1) 和 IBA+ $\operatorname{NAA}(1: 1)$, 朱锦心等 ${ }^{[41]}$ 对紫蓦龙吐珠扞插的研究中也发现较高 IBA 和 NAA 比值的混合液促进生根效果优 于较低 IBA 和 NAA 比值的。王艺等 ${ }^{[42]}$ 和廖伟彪等 ${ }^{[43]}$ 的研究表明外源施加 IAA 的生根效果低于 IBA 处理和 NAA 处理。IBA 在植物体内与葡糖酯结合较慢, 高温下比较稳定, 释放出游离 IBA 较慢, 而 IAA 与天冬氨酸 结合较快, 释放游离 IAA 也快; IAA 易受氧化酶氧化, 而 IBA 不受此酶氧化 ${ }^{[44]}$ 。ABT 生根粉的有效成分主要 是 IAA 和 NAA, 这可能是本研究中 ABT 促进生根效果较差的原因。

前人研究发现, 低浓度激素处理有益于愈伤组织诱导, 中高浓度促进插穗生根及根系生长, 但过高的浓度 则会对插穗组织造成伤害, 影响生根和扦插苗生长 ${ }^{[45]}$ 。吴落军 ${ }^{[19]}$ 对落羽杉 $1 \mathrm{a}$ 生实生苗嫩枝扦插的研究结 果表明,激素浓度对各生根指标具有显著影响, 整体表现为 $200 \mathrm{mg} / \mathrm{L}>150 \mathrm{mg} / \mathrm{L}>100 \mathrm{mg} / \mathrm{L}$, 本实验结果有所 
不同,这可能是本研究的插穗来自于 $9 \mathrm{a}$ 生的落羽杉植株, 其组织老化程度较大,插穗对外源激素敏感度小, 而本研究中设置的浓度梯度过小引起的, 今后还需要扩大激素浓度范围进一步优化实验。激素浸泡时间也是 影响插穗生根的一个重要因素, 在一定范围内, 处理时间越长, 生根效果越好, 但时间过长则可能抑制生 根 ${ }^{[46]}$ 。本研究发现, 随着浸泡时间的增长, 落羽杉插穗的各生根性状大致呈现先升高后降低的趋势, $4 \mathrm{~h}$ 处理 的生根效果优于 $2 \mathrm{~h} 、 6 \mathrm{~h}$ 和 $8 \mathrm{~h}$,张沛健等 ${ }^{[47]}$ 对金蒲桃的研究也发现类似的趋势。

本研究结果表明,外源生长激素对落羽杉扞插生根促进作用, 25 个激素处理组合的生根性状平均隶属函 数值均高于清水对照。本实验中插穗的生根率变幅为 $2.22 \%-31.11 \%$, 黄利斌等 ${ }^{[17]}$ 以 6-7 a 生不同种源的 落羽杉进行嫩枝扞插实验,生根率变幅为 $21.67 \%-75 \%$, 吴落军 ${ }^{[19]}$ 以 $1 \mathrm{a}$ 生落羽杉实生苗的萌生条进行不同 的激素处理,生根率变幅为 $58.26 \%$ - $99.18 \%, 13$ a 生落羽杉不同种源嫩枝扦插的生根率变幅为 $2.60 \%-$ $50 \%$, 可见落羽杉的扞插与母树年龄密切相关。母树年龄越大, 木质化程度越高, 导致插穗含水量和营养物质 含量相对较低,使得插穗生根率下降, 分生组织细胞的活性也受到母树年龄的影响 ${ }^{[48]}$ 。本实验落羽杉嫩枝扦 插生根与其他研究相比生根率较低,究其原因,除了母树年龄不同外,可能与扞插基质、气候等环境原因有关。 此外, 本研究插穗的母树经历了多年反复水淹, 其生长和生理方面发生一定的变化 ${ }^{[49-50]}$, 这可能也是导致实 验差异的原因之一。

本试验对外源激素这一因子进行了初步研究,但从种苗繁育的生产实际来看, 三峡消落带原位生长的落 羽杉插穗生根率和生根质量有待进一步提高, 还需在本研究结果的基础上进行更为系统、全面的研究。另外, 对于三峡消落带原位生长的落羽杉种子繁殖技术方面尚未见报道, 今后可对打破落羽杉种子休眠,促进种子 萌发的措施以及扦插苗与实生苗对于三峡消落带环境的适应性比较开展进一步研究。

\section{4 结论}

皮部生根是大龄落羽杉扦插的主要生根方式。植物生长调节剂的使用对落羽杉插穗生根具有一定的促 进效果,其中外源激素种类对插穗生根的影响最大;其次是处理时间;而外源激素浓度对插穗生根无显著性影 响。通过隶属函数法对 26 个处理组合进行综合比较, 发现 $(\mathrm{IBA}+\mathrm{NAA}) \times 150 \mathrm{mg} / \mathrm{L} \times 4 \mathrm{~h}$ 和 $\mathrm{NAA} \times 250 \mathrm{mg} / \mathrm{L} \times 4$ $\mathrm{h}$ 这两种处理组合效果最好, 是三峡库区消落带落羽杉大龄母树插穗扞插的较佳处理方式。

\section{参考文献 (References) :}

[1 ] 宋虹, 袁中勋, 任庆水, 杨文航, 王朝英, 李昌晓. 三峡消落带落羽杉人工幼林叶片分解及磷释放特征. 生态学报, 2019, 39(21)： 8100-8110.

[ 2 ] 焚大勇, 熊高明, 张爱英, 刘㬢, 谢宗强, 李兆佳. 三峡库区水位调度对消落带生态修复中物种篮选实践的影响. 植物生态学报, 2015, $39(4)$ : 416-432.

[ 3 ] 王业春, 雷波, 张茙. 三峡库区消落带不同水位高程植被和土壤特征差异. 湖泊科学, 2012, 24(2) : 206- 212.

[ 4 ] 孙金伟, 许文盛. 河岸植被缓冲带生态功能及其过滤机理的研究进展. 长江科学院院报, 2017, 34(3): 40-44.

[ 5 ] 李昌晓, 钟章成, 刘芸. 模拟三峡库区消落带土壤水分变化对落羽杉幼苗光合特性的影响. 生态学报, 2005, 25(8): 1953-1959.

[6] 李昌晓, 钟章成. 三峡库区消落带土壤水分变化条件下池杉幼苗光合生理响应的模拟研究. 水生生物学报, 2005, 29(6): 712-716.

[ 7 ] 钟彦, 刘正学, 秦洪文, 熊瑛, 向丽霞, 刘锐, 杨艳, 马䜭. 冬季淹水对柳树生长及恢复生长的影响. 南方农业学报, 2013, 44(2)： 275-279.

[ 8 ] 贾中民, 魏虹, 田晓峰, 李昌晓. 长期水淹对枫杨幼苗光合生理和叶绿素菼光特性的影响. 西南大学学报: 自然科学版, 2009, 31(5): 124- 129 .

[ 9 ] 汪企明, 江泽平, 吕祥生, 张继凡, 鲁开基, 孙永召, 郭士祥, 吴孟军, 陆兴安. 落羽杉属种源研究: 树种生物学特性. 江苏林业科技, 1995, 22(2): 14- 18.

[10] 李昌晓, 耿养会, 叶兵, Rebcca S. 落羽杉与池杉幼苗对多种胁迫环境的响应及其对三峡库区库岸防护林营建的启示. 林业科学, 2010, 46(10): 144- 152 .

[11] Murphy J B, Stanley R G. Increased germination rates of baldcypress and pondcypress seed following treatments affecting the seed coat. Physiologia Plantarum, 1975, 35(2): 135-139.

[12] Liu G D, Li Y C, Hedgepeth M, Wan Y S, Roberts R E. Seed germination enhancement for bald cypress (Taxodium distichum [ L. ] Rich.). Journal of Horticulture and Forestry, 2009, 1(2): 22-26. 
[13] 徐佩贤. 东方杉嫩枝扦插繁殖技术及其生根机理研究 [D ]. 南京: 南京林业大学, 2009 .

[14］杜伟. 桑树硬枝扞插生根的生理生化与分子机理研究 $[D]$. 镇江: 江苏科技大学, 2016.

[15] 李丽娟, 李昌晓, 陈春华, 杨治华, 陈雪梅. 三峡消落带适生植物根系活动调控土壤养分与细菌群落多样性特征. 环境科学, 2020,41 (6) : 2898-2907.

[16] Pezeshki S R, DeLaune R D. Rooting of baldcypress cuttings. New Forests, 1994, 8(4) : 381-386.

[17] 黄利斌, 汪企明, 李晓储, 王伟, 陈志银, 蒋志新, 张继凡, 孙永召, 王永昌, 李伟, 仲伟芹, 陆兴安. 落羽杉属种源研究: 扞插生根能力 变异. 江苏林业科技, 2000, 27(1): 2-7.

[18] Zhou L J. Salt Tolerance, Propagation and Provenance Evaluation of Taxodium as a Landscape and Coastal Wetland Tree[D]. State of Texas: Stephen F. Austin State University, 2007.

[19] 吴落军. 落羽杉的扦插繁殖技术与生根机理研究 $[D]$. 南京: 南京林业大学, 2007.

[20] Creech D L, Zhou L J, Yin Y L. Can Taxodium be improved. Arnoldia, 2011, 69(2) : 11-20.

[21] King A, Arnold M, Welsh D, Watson W. Substrates, wounding, and growth regulator concentrations alter adventitious rooting of baldcypress cuttings. HortScience, 2011, 46(10): 1387-93.

[22] 王书胜, 单文, 张乐华, 杜有新, 李晓花, 王凯红. 基质和 IBA 浓度对云锦杜鹃扦插生根的影响. 林业科学, 2015, 51(9) : 165-172.

[23] 李隆云, 张雪, 杨宪. 灰段毛忍冬扞插生根的解剖学特征研究. 中国中药杂志, 2010, 35(4)：431-434.

[24] 曹凡. 美国山核桃扦插繁殖技术及生根机理研究 [D ]. 南京: 南京林业大学, 2015.

[25] 郭素娟. 林木扦插生根的解剖学及生理学研究进展. 北京林业大学学报, 1997, 19(4): 64-69.

[26] 林艳, 詹亚光, 刘玉喜, 森淑红, 赵志新, 李景云, 李俊涛, 丁毅, 林代斌. 白桦嫩枝扦插不定根形成的解剖观察. 东北林业大学学报, 1996, 24(3) : 15- 19.

[27] 谭健晖. 桉树嫩枝扦插繁殖的研究进展及对策. 广西林业科学, 2003, 32(2) : 74-76, 80-80.

[28］刘勇, 肖德兴, 黄长干, 雷先高. 板栗嫩枝扦插生根解剖学特征研究. 园艺学报, 1997, 24(1): 8- 12 .

[29] Henrique A, Campinhos E N, Ono E O, De Pinho S Z. Effect of plant growth regulators in the rooting of Pinus cuttings. Brazilian Archives of Biology and Technology, 2006, 49(2): 189-196.

[30] Rana R S, Sood K K. Effect of cutting diameter and hormonal application on the propagation of Ficus roxburghii Wall. through branch cuttings. Annals of Forest Research, 2012, 55(1): 69-84.

[31] 孙敬爽, 郑红娟, 贾桂霞, 孙长忠, 文磊. 不同基质、生长调节剂、插穗规格和代谢调节剂对 “蓝星”扞插生根的影响. 北京林业大学学报, 2008, 30(1): 67-73.

[32] Copes D L, Mandel N L. Effects of IBA and NAA treatments on rooting Douglas-fir stem cuttings. New Forests, 2000, 20(3): 249-257.

[33] Zhang W X, Fan J J, Tan Q Q, Zhao M M, Zhou T, Cao F L. The effects of exogenous hormones on rooting process and the activities of key enzymes of Malus hupehensis stem cuttings. PLoS One, 2017, 12(2): e0172320.

[34] Kochhar S, Singh S P, Kochhar V K. Effect of auxins and associated biochemical changes during clonal propagation of the biofuel plant-Jatropha curcas. Biomass and Bioenergy, 2008, 32(12): 1136-1143.

[35] Negishi N, Nakahama K, Urata N, Kojima M, Sakakibara H, Kawaoka A. Hormone level analysis on adventitious root formation in Eucalyptus globulus. New Forests, 2014, 45(4): 577-587.

[36] 贾娟,姚延寿,史敏华,邢金香,郝向春. 生根剂促进槭树植物扦插繁殖的研究进展. 西北林学院学报, 2010, 25(04) : 107-109,134-134.

[37] 孙旭东, 张鹏, 沈海龙. 生根剂和插穗处理对色木械嫩枝扦插生根的影响. 东北林业大学学报, 2018, 46(10): 5-8, 17-17.

[38] 魏黔春. 侧柏古树扦插繁殖技术与生根机理研究 [D]. 北京: 中国林业科学研究院, 2015.

[39] 刘帅. 额济纳旗四种柽柳属树种种子萌发和嫩枝扦插试验研究 [D]. 呼和浩特: 内蒙古农业大学, 2017.

[40］郑健, 郑勇奇, 吴超. 花楸树嫩枝扦插繁殖技术研究. 林业科学研究, 2009, 22(1): 91-97.

[41] 朱锦心, 冯嘉仪, 翁殊斐, 李灿, 罗倩. 不同浓度 IBA、NAA 对紫萼龙吐珠扦插生根的影响. 亚热带植物科学, 2016, 45(3): 285-289.

[42] 王艺, 贾忠奎, 马履一, 邓世崟, 朱仲龙, 桑子阳. 4 种植物生长调节剂对红花玉兰嫩枝扦插生根的影响. 林业科学, 2019, 55(7)： $35-45$.

[43] 廖伟彪, 张美玲, 杨永花, 汉梅兰. 植物生长调节剂浓度和处理时间对月季扦插生根的影响. 甘肃农业大学学报, 2012, 47(3): 47-51.

[44] 张玉臣. 白木香扦插繁殖技术及生根机理研究 [D]. 北京: 中国林业科学研究院, 2010.

[45] 张乐华, 王书胜, 单文, 李晓花, 王凯红, 王兆宏. 基质、激素种类及其浓度对鹿角杜鹃扞插育苗的影响. 林业科学, 2014, 50(3): 45-54.

[46] 陈庆生, 周鹏, 张敏, 黄利斌, 窦全琴. 激素和插穗处理对乌饭树扦插生根的影响. 东北林业大学学报, 2016, 44(4): 41-43, 47-47.

[47］张沛健，高丽琼，尚秀华. 不同激素种类、浓度及浸泡时间对金蒲桃扦插生根的影响. 热带作物学报, 2020, 41(6): 1084-1091.

[48] 杜常健, 孙佳成, 韩振泰, 江泽平, 王金宝, 史胜青. 板栗扞插生根过程的解剖结构和生理变化研究. 西北植物学报, 39(11): 2019, 39 (11) : 1979- 1987.

[49] 贺燕燕, 王朝英, 袁中勋, 李晓雪, 杨文航, 宋虹, 李昌晓. 三峡库区消落带不同水淹强度下池杉与落羽杉的光合生理特性. 生态学报, 2018, 38(8): 2722-2731.

[50］马文超, 刘媛, 周翠, 王婷, 魏虹. 水位变化对三峡库区消落带落羽杉营养特征的影响. 生态学报, 2017, 37(4): 1128-1136. 